\title{
A super-resolution approach for receptive fields estimation of neuronal ensembles
}

\author{
Daniela Pamplona ${ }^{1 *}$, Gerrit Hilgen ${ }^{2}$, Bruno Cessac ${ }^{1}$, Evelyne Sernagor ${ }^{2}$, Pierre Kornprobst ${ }^{1}$ \\ From 24th Annual Computational Neuroscience Meeting: CNS*2015 \\ Prague, Czech Republic. 18-23 July 2015
}

The Spike Triggered Average (STA) is a classical technique to find a discrete approximation of the Receptive Fields (RFs) of sensory neurons [1], a required analysis in most experimental studies. One important parameter of the STA is the spatial resolution of the estimation, corresponding to the size of the blocks of the checkerboard stimulus images. In general, it is experimentally fixed to reach a compromise: If too small, neuronal responses might be too weak thus leading to RF with low Signal-toNoise-Ratio; on the contrary, if too large, small RF will be lost, or not described with enough details, because of the coarse approximation. Other solutions were proposed consisting in starting from a small block size and updating it following the neuron response in a closed-loop to increase its response [2-4]. However, these solutions were designed for single cells and cannot be applied to simultaneous recordings of ensembles of neurons (since each RF has its own size and preferred stimulus). To solve this problem, we introduced a modified checkerboard stimulus where blocks are shifted randomly in space at fixed time steps. This idea is inspired from super-resolution techniques developed in image processing [4]. The main interest is that the block size can be large, enabling strong responses, while the resolution can be finer since it depends on the shift minimum size. In [5] was shown that the STA remains an unbiased RF estimator and, using simulated spike trains from an ensemble of Linear Nonlinear Poisson cascade neurons, it was predicted that this approach improves RF estimation over the neuron ensemble. Here, we test these predictions experimentally on the RFs estimation of 8460 ganglion cells from two mouse retinas, using recordings performed

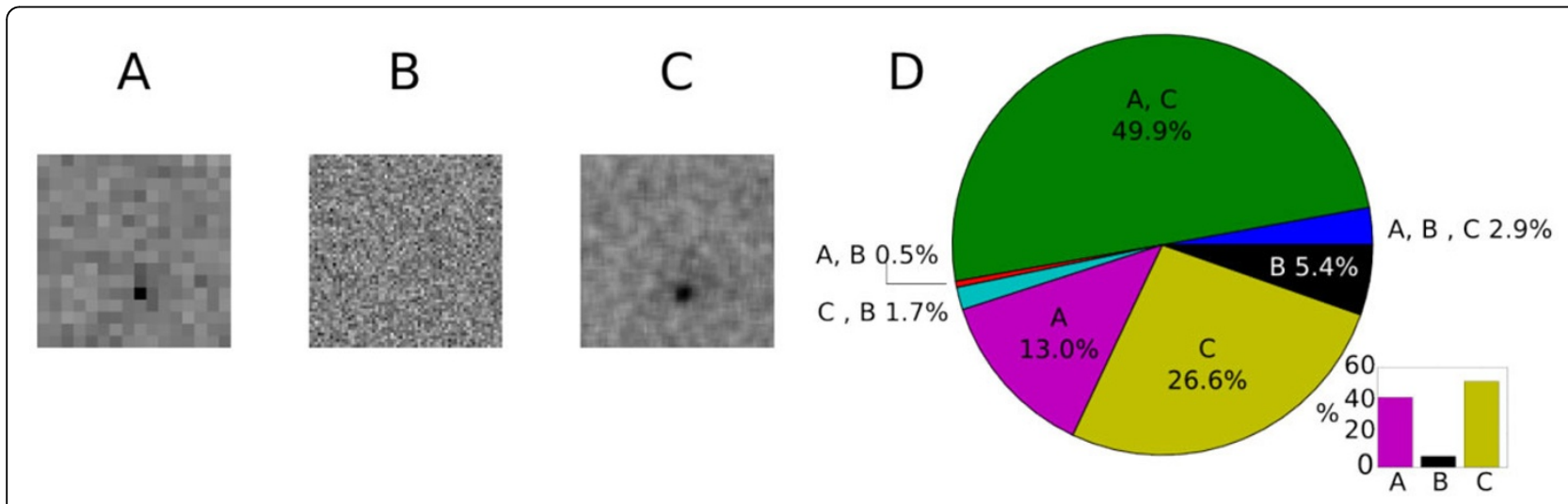

Figure 1 A, B, C: Representative example of STA of one neuron considering stimulus A, B) and C). D: Number of RFs mapped with each stimulus.

\footnotetext{
* Correspondence: daniela.pamplona@inria.fr

'INRIA Sophia Antipolis Méditerranée, Neuromathcomp, 2004 Route des

Lucioles, 06902 Valbonne, France

Full list of author information is available at the end of the article
} 
with a large scale high-density multielectrode array. To illustrate the main interest of the approach, in Figure 1 we show a representative example of STA for one neuron where RFs have been obtained using the three following stimuli (all presented during $15 \mathrm{~min}$, for one retina displayed at $10 \mathrm{~Hz}$, for the other at $30 \mathrm{~Hz}$ ): (A) standard checkerboard stimulus with block size of $160 \mu \mathrm{m}$, (B) standard checkerboard stimulus with block size of $40 \mu \mathrm{m},(\mathrm{C})$ checkerboard stimulus with block size of $160 \mu \mathrm{m}$ and arbitrary shifts of $40 \mu \mathrm{m}$ in $\times$ and $y$-directions. Results show spatial resolution can be improved in case $(C)$, while nothing could be obtained in (B) by changing only the block size of the standard stimulus. At the population level, plot (D) shows the number of the RFs that could be recovered for each stimuli, using a decision criteria based of the RFs value distribution. Most of the RFs were mapped with both methods (A) and (C) (49.9\%). However, the proposed case $(\mathrm{C})$ allows to recover $51 \%$ of the mapped RFs at a resolution of $40 \mu \mathrm{m}$, while in the classical case (A), $41 \%$ of the RFs could be found at a resolution of only $160 \mu \mathrm{m}$. Thus, the method does improve the quality of the RF estimation and the amount of successfully mapped RFs in neural ensembles.

\section{Acknowledgements}

This work was partially supported by the EC IP project FP7-ICT-2011-9 no. 600847 (RENVISION)

\section{Authors' details}

INRIA Sophia Antipolis Méditerranée, Neuromathcomp, 2004 Route des Lucioles, 06902 Valbonne, France. ${ }^{2}$ Institute of Neuroscience, Faculty of Medical Sciences, Framlington Place, Newcastle upon Tyne, NE2 4HH.

Published: 18 December 2015

\section{References}

1. Chichilnisky E J: A simple white noise analysis of neuronal light responses. Network 2001, 12:199-213.

2. Foldiak P: Stimulus optimization in primary visual cortex. Neurocomputing 2001, 38-40:1217-1222

3. Machens CK: Adaptive sampling by information maximization. Phys Rev Lett 2002, 88(22):228104.

4. MacKay D: Information-based objective functions for active data selection. Neural Comput 1992, 4:590-604.

5. Nasrollahi K, Moeslund T B: Super-resolution: a comprehensive survey. Mach Vis Appl 2014, 25:1423-1468.

6. Cessac B, Kornprobst P: Shifting stimulus for faster receptive fields estimation of ensembles of neurons. Cosyne Abstracts. Salt Lake City USA 2015.

doi:10.1186/1471-2202-16-S1-P130

Cite this article as: Pamplona et al:: A super-resolution approach for receptive fields estimation of neuronal ensembles. BMC Neuroscience 2015 16(Suppl 1):P130.

\section{Submit your next manuscript to BioMed Central} and take full advantage of:

- Convenient online submission

- Thorough peer review

- No space constraints or color figure charges

- Immediate publication on acceptance

- Inclusion in PubMed, CAS, Scopus and Google Scholar

- Research which is freely available for redistribution

Submit your manuscript at www.biomedcentral.com/submit
C Biomed Central 